\title{
Improved Performance in Raman-based Distributed Temperature Sensing with coded OTDR and Discrete Raman Amplification
}

\author{
G. Bolognini ${ }^{1}$, J. Park ${ }^{2}$, A. Chiuchiarelli ${ }^{3}$, N. Park ${ }^{2}$, and F. Di Pasquale ${ }^{1}$ \\ 1: Scuola Superiore Sant'Anna, via G. Moruzzi 1, 56124 Pisa, Italy.E-mail: gabriele.bolognini@cnit.it \\ 2: School of EECS, Seoul National University, Korea. E-mail: nkpark@plaza.snu.ac.kr \\ 3:Photonic Networks National Laboratory, CNIT, Pisa, Italy.
}

\begin{abstract}
Using 255-bit Simplex-coded OTDR we demonstrate enhanced performance in Raman-based DTS (30 km with $5 \mathrm{~K}$ and $17 \mathrm{~m}$ resolution) using low-power $(80 \mathrm{~mW})$ laser-diodes. Additional discrete Raman amplification of coded pulses allows more than $40 \mathrm{~km}$ measurement range.

(C) 2005 Optical Society of America

OCIS codes: $060.2370,290.5860$
\end{abstract}

\section{INTRODUCTION}

Raman based distributed temperature sensors (DTS) have attracted a great deal of attention in recent years, due to their wide range of possible practical applications [1]. In most schemes the ratio of temperature dependent backscattered spontaneous Raman scattering (SRS) to Raleigh backscattered pump intensity is monitored through optical time-domain reflectometry (OTDR) techniques, providing accurate location of temperature variations along the fiber length.

The development and commercial exploitation of such DTS systems have been however hindered by the low backscattered intensity of the Anti-Stokes light, which requires high peak power levels in the OTDR, as well as high sensitivity detection schemes, in order to make the sensor performance attractive. These features have made other DTS systems more attractive and cost-effective, such as spontaneous Brillouin scattering based distributed sensors [2]. We have recently shown that coded OTDR techniques allows for enhanced performance in Raman based DTS, avoiding the use of high peak power sources; in particular, using 63 bit simplex OTDR coding and low-power (80 $\mathrm{mW}$ ) commercially available laser diodes, we have demonstrated a performance improvement in measurement range higher than $12 \mathrm{~km}$ with $15 \mathrm{~m} / 5 \mathrm{~K}$ spatial/temperature resolution [3] with respect to conventional OTDR; this was achieved using off-shell conventional OTDR hardware and standard $3^{\text {rd }}$-window optical communication components, providing then a cost-effective solution.

In this paper, using 255-bit Simplex codes, and dispersion shifted (DS) sensing fibers, characterized by higher Rayleigh back-scattering coefficients, we enhance the sensing range up to $30 \mathrm{~km}$ with $17 \mathrm{~m} / 5 \mathrm{~K}$ spatial/ temperature resolution. Moreover, by amplifying the coded OTDR signal at $1550 \mathrm{~nm}$ before launching it into the sensing fiber, we demonstrate a sensing range greater than $40 \mathrm{~km}$ with same spatial/temperature resolution $(17 \mathrm{~m} / 5 \mathrm{~K})$.

As standard Erbium-doped fiber amplifiers and counter-pumped discrete Raman amplifiers can not be effectively used for coded OTDR amplification, due to undesirable waveform distortion related to slow transient effects, we have developed a co-pumped low-noise discrete Raman amplifier based on DS fiber, which provides up to $6 \mathrm{~dB}$ net gain to the coded OTDR signal. Standard fiber Raman laser (FRL) technology at $1460 \mathrm{~nm}$ is used to co-pump the discrete Raman amplifier; no penalties associated to pump to OTDR signal noise transfer have been observed, due to limited bandwidth of the electrical receiver.

\section{THEORY}

In OTDR based DTS systems a short laser pulse is sent along the sensing fiber and the backscattered Raman light, containing information about loss and temperature along the fiber, is detected with high temporal resolution. In particular, as the Anti-Stokes line depends on both fiber temperature, due to changes in phonon distribution, and fiber loss, the ratios of Anti-Stokes to Stokes light intensities or Anti-Stokes to backscattered pump light intensities are typically used for effectively distinguishing distributed temperature variations from local losses.

In our measurements we use the ratio between the backward propagating Anti-Stokes intensity $I_{A S}$ and the backscattered pump intensity $I_{B S}$, which can be approximately expressed as: 


\section{ThE45.pdf}

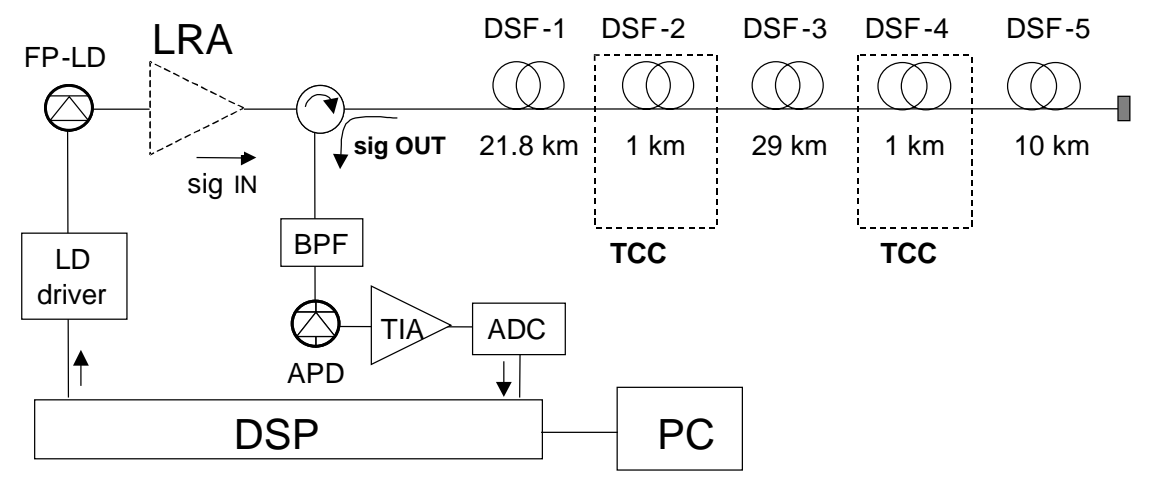

Fig.1. Experimental set-up implementing Raman-based DTS with coded OTDR

$\frac{I_{A S}}{I_{B S}} \propto\left[\exp \left(\frac{h \Delta v_{R}}{k T}\right)-1\right]^{-1}$

where $h$ is the Planck constant, $k$ is the Boltzmann constant, $T$ is the absolute temperature, and $\Delta v_{\mathrm{R}}$ is the separation between Raman Anti-Stokes and pump light frequencies.

Equation (1) is properly integrated along the distance and spectrum to take into account for the wavelength dependent loss along the light path and the cross-section spectrum of Raman Anti-Stokes scattering; it allows the measurement of the temperature variation along the whole optical fiber with respect to the known absolute temperature $(300 \mathrm{~K})$ at the fiber input.

Coding techniques in OTDR schemes provide substantial SNR improvements, which can be quantified by the coding gain, defined as the ratio of SNR obtained with coded OTDR to SNR obtained with conventional ODTR at a given distance, and equal total number of measured traces. For Simplex coding, used in this work, the coding gain $G_{c o d}$ for an arbitrary code length $L$ is given by [4]: $G_{C O D}=(L+1) / 2 \sqrt{L}$.

\section{EXPERIMENT}

Figure 1 shows the experimental set-up used for implementing the Raman-based DTS system based on coded OTDR and lumped Raman amplification of OTDR coded pulses. An in-house-built PC-controlled OTDR board with a digital signal processor (DSP) was used to intensity-modulate the laser diode at $1550 \mathrm{~nm}$ according to the pulse patterns of Simplex coding [5], with 100 ns single bit pulse-width, as well as for implementing single pulses in conventional OTDR.

The light source was a commercially available Fabry-Perot laser diode (FP-LD centered at $1550 \mathrm{~nm}, 80 \mathrm{~mW}$ output power, $10 \mathrm{~nm}$ FWHM, thus preventing coherent speckles). The input pulses were injected into the sensing fiber through an optical circulator, and the backscattered lightwave signals were then coupled to the receiver after a largebandwidth band-pass filter (bandwidths are 1400-1510 nm for Anti-Stokes light and 1520-1600 nm for Rayleighscattered pump light).

In this paper we compare the performance of standard OTDR, 255 bit S-coded OTDR and 255 bit S-coded OTDR combined with a discrete (or lumped) Raman amplification scheme (LRA), whose structure is schematically shown in Fig. 2, to provide low-noise optical amplification for the coded pulse pattern at $1550 \mathrm{~nm}$, as explained below.

The receiver, described in Fig. 1 (with improved performance with respect to [3]), is based on a high sensitivity InGaAs avalanche photodiode (APD), amplified by a high gain transimpedance amplifier (TIA), with about $3 \mathrm{MHz}$ bandwidth. The estimated temperature sensitivity with this set-up was about $0.65 \% / \mathrm{K}$ (at T=300 K).

After the receiver block, an analog-to-digital converter (ADC) was used to sample the incoming analog data trace at $20 \mathrm{MHz}$. The code-imprinted traces were hence transmitted to the PC, where the de-coding process was carried out. The spatial resolution (defined by the measured 10\%-90\% response time) for the current DTS system was found to

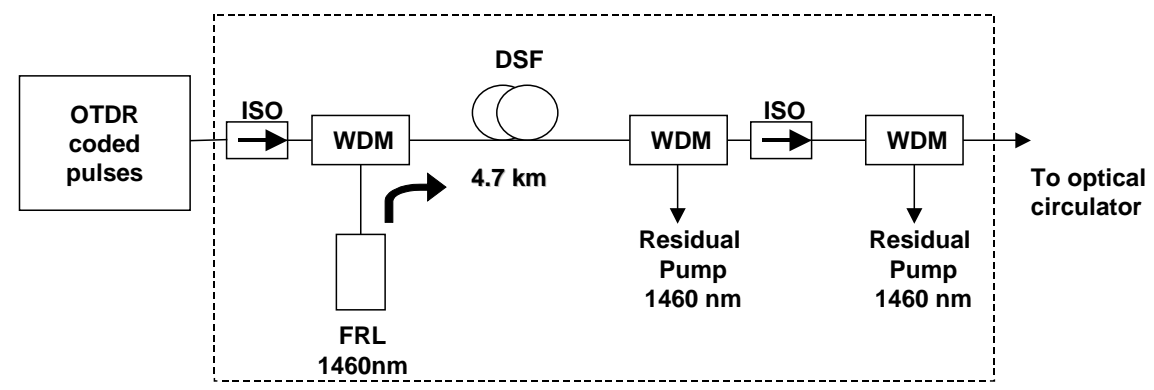

Fig. 2. Scheme of co-pumped lumped Raman amplifier scheme used to amplify long coded pulse sequences. 


\section{ThE45.pdf}
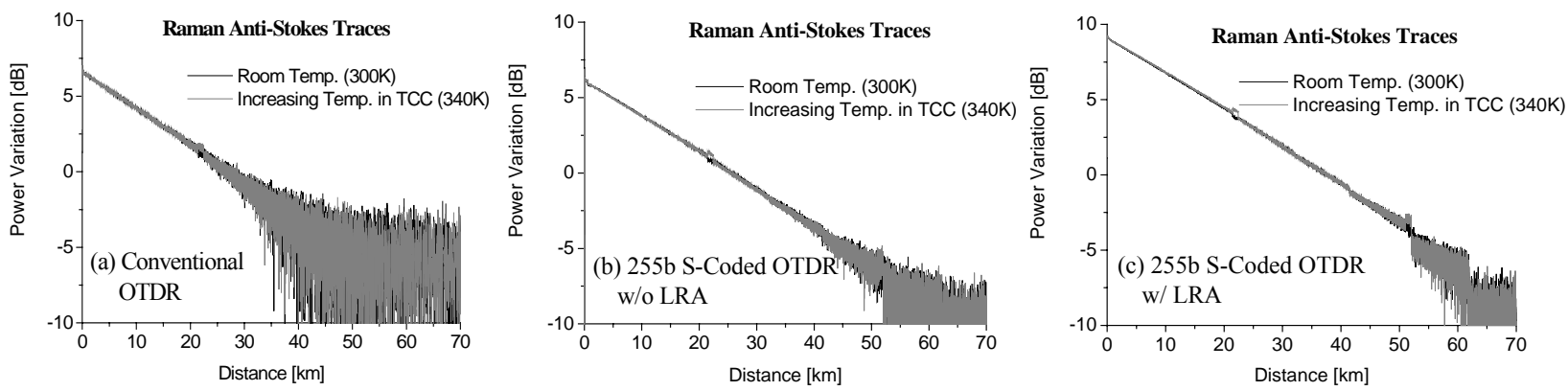

Fig. 3. Traces of received Anti-Stokes light power obtained using (a) conventional OTDR, (b) coded OTDR (255 bit Simplex coding), and (c) coded OTDR and lumped Raman amplification.

be $17 \mathrm{~m}$, mainly limited by the TIA bandwidth. Five spools of fibers (DSF-1 to DSF-5) have been used (lengths are $21.8,1,29,1$, and $10 \mathrm{~km}$ respectively), which have been spliced together to compose a total of $62.8 \mathrm{~km}$ sensing fiber link. DSF-2 and DSF-4 have been put inside a temperature-controlled chamber (TCC), while the other spools were kept at room temperature $(300 \mathrm{~K})$.

Regarding optical amplification of coded pulses from LD, note that the LRA shown in Fig. 2 is based on a copumping scheme; actually, the use of other amplification techniques, such as EDFA or counter-pumped LRA would not be effective due to undesirable waveform distortions of the coded OTDR signal at $1550 \mathrm{~nm}$, induced by power transient excursions [6] arising due to long codeword duration (about $25 \mu$ s for a 255-bit codeword).

In the scheme of Fig. 2, a WDM combiner couples in co-propagating direction the coded pulses from FP-LD (1550 $\left.\mathrm{nm}, \mathrm{P}_{\text {peak }}=80 \mathrm{~mW}\right)$ together with a FRL pump $\left(1460 \mathrm{~nm}, \mathrm{P}_{\max }=1.9 \mathrm{~W}\right)$, into a DSF spool $\left(4.7 \mathrm{~km}, \lambda_{0}=1540 \mathrm{~nm}\right)$. At the LRA output, two cascaded WDM combiners have been used to separate the OTDR pulses from the residual pump power with high directivity, to avoid leakage of FRL light into the sensing fiber.

The LRA stage provides a net gain of about $6 \mathrm{~dB}$, thus allowing one to attain about $320 \mathrm{~mW}$ peak power in coded pulse patterns, with negligible power excursions within one codeword (note that, in spite of the long codeword duration, peak power remains below the threshold value for onset of detrimental nonlinear effects [7], thus allowing for spontaneous Raman temperature sensing).

Note that the use of DS fibers in LRA is crucial in obtaining optical amplification with negligible power excursion within one codeword, because the small chromatic dispersion midway between pump and signal wavelengths results in a small walk-off during amplification with respect to bit duration, and negligible transient effects due to crossgain saturation from neighboring bits (total walk-off at LRA end has been calculated to be about $1.5 \mathrm{~ns}$ ).

Also, the high relative intensity noise value of standard FRL technology is not an issue in these applications due to the limited receiver bandwidth (few $\mathrm{MHz}$ ), contrarily to what happens in optical transmission at higher bit rates, where co-pumping with FRL is strongly affected by intensity noise transfer .

\section{RESULTS}

Fig. 3 reports the obtained OTDR traces for Anti-Stokes light with all sensing fiber spools kept at room temperature $(300 \mathrm{~K}$, black line), and with the two spools DSF-2 and DSF-4 heated at $340 \mathrm{~K}$ (gray line), showing the expected increase in Anti-Stokes intensity for the heated fiber spools. Fig. 3(a) reports the curves obtained with conventional OTDR and 180,000 time averages, Fig. 3(b) shows the traces obtained using 255 bit S-coded OTDR (80 mW peak power at sensing fiber input), and Fig. 3(c) the same traces in case of 255 bit S-coded OTDR and additional discrete Raman amplification of the OTDR signal (320 $\mathrm{mW}$ peak power at sensing fiber input).

Note that for a sensible comparison of standard and coded OTDR techniques, a number of 706 time averaging has been used for a single codeword ( 255 orthogonal codewords are used), thus giving the same total number of 180,000 acquired traces as with conventional OTDR.
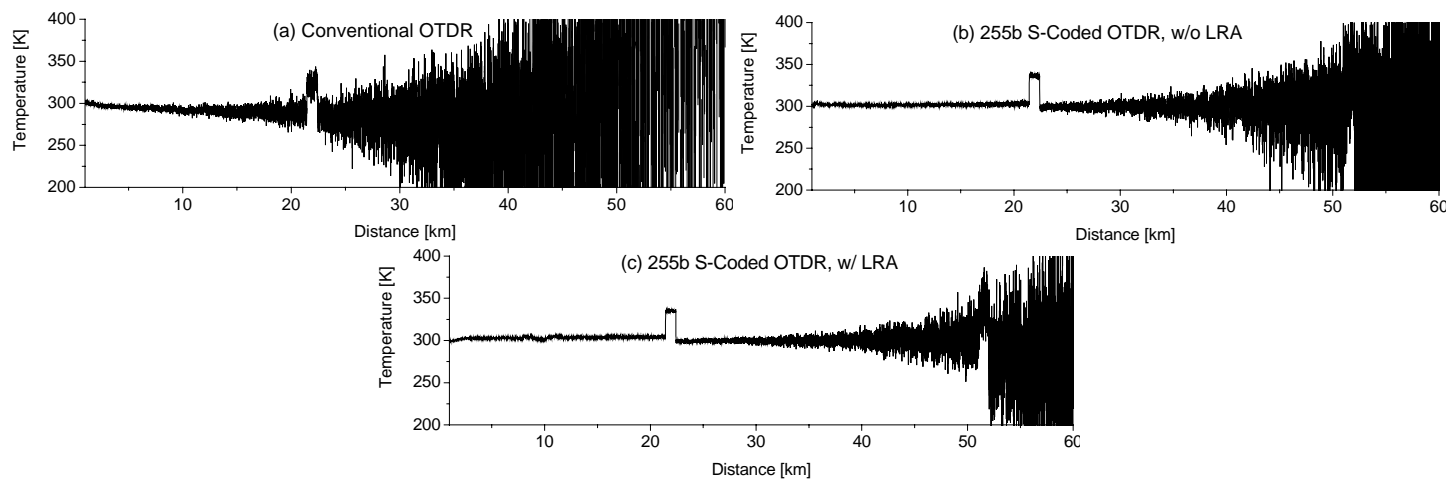

Fig. 4 Temperature distribution versus fiber length obtained from measured traces using (a) conventional OTDR, (b) coded OTDR (255 bit Simplex codes), and (c) coded OTDR and lumped Raman amplification (DSF-2 and DSF-4 spools have been heated at $340 \mathrm{~K}$ ). 


\section{ThE45.pdf}

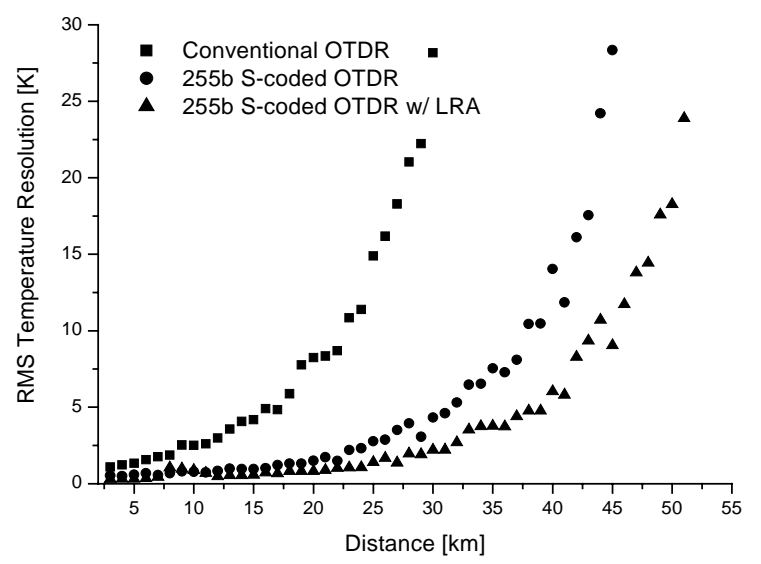

Fig. 5. RMS temperature resolution vs distance using conventional OTDR (squares), coded OTDR (circles), and coded OTDR with lumped Raman amplification (triangles).

It can be seen in Fig. 3 how coded OTDR allows for a sensible increase in SNR with respect to uncoded OTDR (for same input power and same total number of acquired traces), hence allowing for an increased measurement range of temperature sensing. Also evident is how discrete Raman amplification of the coded OTDR signal allows for higher launched power and consequent improved SNR.

The temperature distribution calculated from the Anti-Stokes to Rayleigh backscattering ratio is shown in Fig. 4(a), 4(b) and 4(c) using, respectively, conventional OTDR, pulse coding, and pulse coding together with discrete Raman amplification. The OTDR trace of Rayleigh backscattering (not shown), thanks to its higher intensity levels, was acquired using conventional OTDR only, allowing for adequate SNR values at all distances from fiber near-end. The ratio $\mathrm{I}_{\mathrm{AS}} / \mathrm{I}_{\mathrm{BS}}$, corrected for wavelength dependent losses, has been used for temperature assessing.

It is evident from Fig. 4 that temperature distribution by using 255 bit coding allows for more accurate temperature assessing and increased measurement distance at same SNR with respect to conventional OTDR. The additional discrete optical amplification is seen from Fig. 4(c) to allow for further extension of the sensing range with a given resolution, allowing, e.g., to detect temperature variation in DSF-4 heated fiber spool, placed at distance of $50 \mathrm{~km}$.

Achieved RMS temperature resolution versus distance is reported in Fig. 5 showing a curve for each of the three used DTS techniques, namely conventional OTDR (squares), Simplex-coded OTDR (circles), and Simplex-coded OTDR is association with lumped Raman amplification (triangles). For a desired temperature resolution, the measurement range is greatly enhanced by the S-coding and discrete Raman amplification.

In particular, by considering a reference temperature resolution of $5 \mathrm{~K}$, we can see that the sensing range is limited to less than $15 \mathrm{~km}$ with conventional OTDR, while 255-bit Simplex-coded OTDR allows for about $30 \mathrm{~km}$ measurement range (i.e. $15 \mathrm{~km}$ increase); providing discrete Raman amplification to the coded OTDR signal allows a measurement range greater than $40 \mathrm{~km}$ (i.e. $10 \mathrm{~km}$ further increase). These experimental results are consistent with theoretical predictions based on the coding gain formula provided in Sec. 2. Also the net gain $(6 \mathrm{~dB})$ in LRA well explains the sensing range enhancement $(10 \mathrm{~km})$, pointed out in Fig. 5 when combing S-coding and discrete Raman amplification.

\section{CONCLUSION}

In conclusion, improved performance in Raman based DTS systems has been demonstrated using 255 bit Simplex coded OTDR, combined with discrete Raman amplification. Experimental results show that 255 bit Simplex coded OTDR provides a sensing range enhancement, with respect to conventional OTDR, of about $15 \mathrm{~km}$ with $17 \mathrm{~m} / 5 \mathrm{~K}$ spatial/ temperature resolution, making the use of low power $(80 \mathrm{~mW})$ commercially available laser diodes practical for most applications. Further performance improvement can be achieved introducing low-noise discrete Raman. amplification of coded pulses, allowing more than $40 \mathrm{~km}$ measurement range with same spatial/temperature resolution $(17 \mathrm{~m} / 5 \mathrm{~K})$.

\section{REFERENCES}

1. M. Ahangrani et al., "Spontaneous Raman scattering in optical fibers with modulated probe light for distributed temperature Raman remote sensing", J. Lightwave Technol., 17, 1379-1391 (1999).

2. Y. T. Cho et al.," $50-\mathrm{km}$ single-ended spontaneous-Brillouin-based distributed-temperature sensor exploiting pulsed Raman amplification”, Opt. Lett., 28, 1651 (2005).

3. G. Bolognini et al., "Performance enhancement of Raman-based distributed temperature sensors using Simplex codes", OFC Conf. Tech. Dig., 2006, paper OtuL1.

4. D. Lee et al., "Analysis and experimental demonstration of simplex coding technique for SNR enhancement of OTDR ," LTIMC-15, Proceedings IEEE LTIMC, New York, October 2004.

5. M. D. Jones, "Using simplex codes to improve OTDR sensitivity", IEEE Photon. Technol. Lett., 15, 822-824 (1993).

6. G. Bolognini et al., "Transient effects in gain clamped discrete Raman amplifiers", IEEE Photon. Technol. Lett., 16, 66-68 (2004)

7. P. C. Wait et al., "A theoretical comparison of spontaneous Raman and Brillouin based fibre optic distributed temperature sensors", Opt. Comm., 144, 17-23 (1997). 